\title{
Contralateral mastectomy and survival after breast cancer in carriers of BRCA1 and BRCA2 mutations: retrospective analysis
}

\begin{abstract}
Objective To compare the survival rates of women with BRCA associated breast cancer who did and did not undergo mastectomy of the contralateral breast.

Design Retrospective analysis.

Setting 12 cancer genetics clinics.

Participants 390 women with a family history of stage I or II breast cancer who were carriers of BRCA1 and BRCA2 mutations and initially treated with unilateral or bilateral mastectomy. 181 patients had mastectomy of the contralateral breast. Patients were followed for up to 20 years from diagnosis.

Main outcome measure Death from breast cancer.

Results 79 women died of breast cancer in the follow-up period (18 in the bilateral mastectomy group and 61 in the unilateral mastectomy group). The median follow-up time was 14.3 years (range $0.1-20.0$ years). At 20 years the survival rate for women who had mastectomy of the contralateral breast was $88 \%$ (95\% confidence interval $83 \%$ to $93 \%$ ) and for those who did not was $66 \%$ (59\% to $73 \%$ ). In a multivariable analysis, controlling for age at diagnosis, year of diagnosis, treatment, and other prognostic features, contralateral mastectomy was associated with a $48 \%$ reduction in death from breast cancer (hazard ratio 0.52 , $95 \%$ confidence interval 0.29 to $0.93 ; P=0.03$ ). In a propensity score adjusted analysis of 79 matched pairs, the association was not significant $(0.60,0.34$ to $1.06 ; P=0.08)$. Based on these results, we predict that of 100 women treated with contralateral mastectomy, 87 will be alive at 20 years compared with 66 of 100 women treated with unilateral mastectomy.
\end{abstract}

Conclusions This study suggests that women who are positive for BRCA mutations and who are treated for stage I or II breast cancer with bilateral mastectomy are less likely to die from breast cancer than women who are treated with unilateral mastectomy. Given the small number of events in this cohort, further research is required to confirm these findings.

\section{Introduction}

Women who carry a germline mutation in either the BRCA 1 or BRCA2 gene have a lifetime risk of breast cancer of $60-70 \%$, and once diagnosed as having breast cancer, have a high risk of a second primary breast cancer. ${ }^{2}{ }^{3}$ The principal goal of treating hereditary breast cancer is to minimise the likelihood of patients dying from a first breast cancer, but it is also important to minimise the incidence of, and mortality from, a second primary cancer. Traditionally, breast cancer trialists and clinical epidemiologists focus their attention on the 10 year period after diagnosis, because this is when the majority of cancer related deaths occur. However, a mortality benefit from preventing a second primary breast cancer is unlikely to be apparent within this narrow interval, given that second primary cancers accumulate slowly and for an extended period. ${ }^{24}$ Little information is available on the long term survival experience of women with a BRCA1 or BRCA2 mutation who are treated for breast cancer. Most previous studies involve a small number of participants or follow them for a short period, and no previous study has looked at mortality in association with mastectomy of the contralateral breast..$^{5-8}$ In North America, approximately half of women with a BRCA mutation will undergo mastectomy 
of the contralateral breast to prevent a second breast cancer, ${ }^{3}$ but it has not yet been shown that contralateral mastectomy reduces breast cancer related mortality. ${ }^{9}$

We reviewed the 20 year survival experience of 390 women with early stage breast cancer, diagnosed from 1975 to 2009, who are known carriers or likely to carry a deleterious mutation in the BRCA1 or BRCA2 gene and were treated with unilateral or bilateral mastectomy.

\section{Methods}

To identify trial participants we reviewed the family histories of cancer for those who received genetic counselling at 12 participating clinics specialising in cancer genetics. We considered a family to be eligible for the study when a BRCA1 or BRCA2 mutation was documented in the family and at least one case of invasive breast cancer was recorded. Eligible participants included all women from these families with a diagnosis of stage I or stage II breast cancer at age 65 or less, between 1975 and 2008. We considered for eligibility those women who were alive or had died but we excluded those with a previous diagnosis of cancer (including breast cancer) or those who resided outside of North America. To be included in the study it was not necessary to be proved a carrier of the family mutation; however, we also excluded affected women who were known to be non-carriers.

We identified 615 families, totalling 1773 cases of breast cancer (fig $1 \Downarrow$ ). Of these 1773 cases of breast cancer, we excluded 417 women because their diagnosis was before 1975 and 70 women because they were aged more than 65 at diagnosis. An additional 29 women were known not to carry the familial mutation and were therefore excluded, along with 19 women because they had a diagnosis of other cancer before breast cancer and 26 women because they were treated outside of North America.

Of the remaining 1212 women, we were able to obtain identifying information for 927 (76.5\%). We attempted to contact each woman or her next of kin to obtain permission to review the medical records. Nineteen women (or their next of kin) refused to provide consent for the release of medical records. We requested the medical records for the remaining 908 women from the hospital where they received treatment. In 76 cases the hospital was not able to locate the record or did not forward the requested documents, therefore we obtained the medical records for 832 women $(91.6 \%)$.

After review of the medical records, we excluded an additional 441 women. Of these, we excluded 32 women because the tumour stage was more than 2, 18 because the tumour was non-invasive (ductal carcinoma in situ or lobular carcinoma in situ), and two because they refused treatment. We also excluded 20 women who had an initial diagnosis of bilateral breast cancer. As we wished to compare women treated with unilateral mastectomy with those treated with bilateral mastectomy, we excluded 370 women who were treated with breast conserving surgery. The remaining 390 women are included in the analysis. Of the 390 women, 336 were proved to be gene carriers (86\%) and $54(14 \%)$ were not tested but were from families with a BRCA mutation and were therefore likely to be gene carriers. The 390 women were from 290 different families.

\section{Study protocol}

We reviewed the medical treatment records and pathology documents and recorded tumour size (in centimetres), nodal status (positive or negative), and tumour grade (I to III). Oestrogen receptor status was recorded as positive, negative, equivocal, or unknown. We recorded the use of chemotherapy (yes or no), tamoxifen (yes or no), and radiotherapy (yes or no). We established whether or not the patient had undergone a bilateral oophorectomy and, if so, the date of the operation. We recorded whether the initial surgery was lumpectomy, unilateral mastectomy, or bilateral mastectomy, and included only those women who were initially treated with unilateral or bilateral mastectomy. From the medical record we abstracted causes of death and recorded these as breast cancer, ovarian cancer, another cancer, another cause, or unknown. We updated the vital status of the cohort every two years. A single trained data abstractor coded and entered all data.

\section{Statistical analysis}

In all analyses the primary endpoint was death from breast cancer. For women with bilateral breast cancer it was not possible to distinguish death due to a first primary cancer from that of a second primary breast cancer. We considered women to be at risk for death from the date of the first surgical procedure until the last date of follow-up or until death from breast cancer, death from another cause, or the date of last follow-up. To estimate hazard ratios we used the Cox proportional hazards model, implemented in SAS. The principal exposure was contralateral mastectomy. In some cases, bilateral mastectomy was performed as the initial surgery and in other cases contralateral mastectomy was performed as a second surgery at some time after the initial (unilateral) mastectomy. Therefore, we treated contralateral mastectomy as a time dependent variable. In a multivariable analysis we estimated the hazard ratio associated with contralateral mastectomy on survival, adjusting for the use of tamoxifen, chemotherapy, and radiotherapy (as dichotomous variables) and for oophorectomy (as a time dependent variable). We adjusted the hazard ratios for mutation status (BRCA1 versus BRCA2), age at diagnosis (years), year of diagnosis, tumour size $(\mathrm{cm})$, and lymph node status (positive or negative). In addition, we did a separate analysis using a matched propensity score approach. We generated a propensity score, based on gene (BRCA1 or BRCA2), tumour size, nodal status, age at diagnosis, year of diagnosis, radiotherapy (yes or no), tamoxifen (yes or no), and chemotherapy (yes or no). The maximum differences in propensity score between case and matched control was set at 0.02 . We generated 79 matched pairs. The mean propensity score was 0.603 for controls and 0.618 for cases.

\section{Results}

Table $1 \Downarrow$ presents the characteristics of the 390 women with breast cancer and their treatments. The women were given a diagnosis between 1977 and 2009 and were followed from diagnosis for a mean of 13.0 years (range 0.1-20.0 years), until June 2012. Overall, 79 participants (20\%) died of breast cancer during the follow-up period; the mean time to death from diagnosis was 7.1 years (range $0.7-19.3$ years).

Of the 390 participants, 44 were initially treated with bilateral mastectomy and 346 were initially treated with unilateral mastectomy. Of those who were treated with unilateral mastectomy, 137 went on to have mastectomy of the contralateral breast at a later date. The average time from diagnosis to contralateral mastectomy was 2.3 years. Table $2 \Downarrow$ compares the women with unilateral and bilateral mastectomies. To evaluate the impact on breast cancer specific survival, contralateral mastectomy was treated as a time dependent covariate. The unadjusted hazard ratio for the women treated with contralateral mastectomy compared with unilateral 
mastectomy was 0.48 (95\% confidence interval 0.29 to 0.82 ; $\mathrm{P}=0.007)$ for the entire 20 year study period and 0.20 ( 0.05 to $0.85 ; \mathrm{P}=0.03$ ) for the second decade of follow-up (table $3 \Downarrow$ ). The adjusted hazard ratio for the women treated with contralateral mastectomy compared with unilateral mastectomy was $0.52(0.29$ to $0.93 ; \mathrm{P}=0.03)$ for the entire study period and 0.20 ( 0.05 to $0.89 ; \mathrm{P}=0.03)$ for the second decade of follow-up. In the propensity score adjusted analysis, which included 79 matched pairs, the association was not as strong and was no longer statistically significant (hazard ratio $0.60,95 \%$ confidence interval 0.34 to $1.06 ; \mathrm{P}=0.08$ ). For all cause mortality, the adjusted hazard ratio for the women treated with contralateral (bilateral) mastectomy compared with unilateral mastectomy was 0.58 ( 0.34 to $0.97 ; \mathrm{P}=0.04)$ for the entire study period and 0.36 ( 0.13 to $0.96 ; \mathrm{P}=0.04$ ) for the second decade of follow-up. The association between contralateral mastectomy and death from breast cancer in the first 10 years from diagnosis was not statistically significant in either the univariable or multivariable analysis.

Overall, 54 of the 390 women in the study were assumed to carry a mutation but were not tested. We repeated the analysis after removing these 54 women. The adjusted hazard ratio for the women treated with contralateral (bilateral) mastectomy compared with unilateral mastectomy was 0.55 (0.27 to 1.13 ; $\mathrm{P}=0.10)$ for the entire study period and 0.19 (0.04 to 0.84 ; $\mathrm{P}=0.03$ ) for the second decade of follow-up.

The majority of the contralateral mastectomies (94\%) were performed in the first 10 years of follow-up. Of the 20 women who were alive at 10 years, but died of breast cancer between years 10 and 20 , only two $(10 \%)$ had a previous contralateral mastectomy, compared with $43 \%$ (108 of 251) of women who were followed for at least 10 years and who were alive at the end of the follow-up period ( $\mathrm{P}=0.005$ ) (fig $2 \Downarrow$ ).

To estimate the impact of a diagnosis of breast cancer in the contralateral breast on mortality, contralateral breast cancer was treated as a time dependent covariate. In this analysis, after a diagnosis of breast cancer in the contralateral breast, the mortality rate was increased twofold (hazard ratio $2.17,95 \%$ confidence interval 1.26 to $3.75 ; \mathrm{P}=0.005)$. Of the 20 women who died between years 10 and 20, $11(55 \%)$ had experienced a second primary cancer (three ipsilateral, nine contralateral). By contrast, of the 231 women who were followed for at least 10 years and who were alive at last follow-up, $61(26 \%)$ had experienced a second primary cancer (13 ipsilateral, 50 contralateral) ( $\mathrm{P}=0.007$ for difference). One woman had a diagnosis of breast cancer in the contralateral breast after contralateral subcutaneous mastectomy, diagnosed three years after the initial breast cancer diagnosis and bilateral mastectomy. She presented with a $1 \mathrm{~cm}$ mass in the pectoralis muscle and was alive 20 years later.

Table $4 \Downarrow$ presents the hazard ratios associated with other clinical and prognostic factors in the patient cohort. Chemotherapy and oophorectomy were both associated with reductions in mortality in the multivariable analysis, but neither of these associations was statistically significant. The 20 year survival for carriers of the BRCA1 mutation was $72.2 \%$ and for carriers of the BRCA2 mutation was $78.1 \%(\mathrm{P}=0.07 \log$ rank test). In the adjusted analysis, the survival experience of carriers of the BRCA2 mutation was better than that of carriers of the BRCA1 mutation (hazard ratio $0.45,95 \%$ confidence interval 0.26 to 0.81 ), but among patients treated with chemotherapy, the survival advantage for carriers of the BRCA2 mutation was attenuated $(0.62,0.30$ to 1.31$)$.

\section{Discussion}

In two previous studies of this cohort of women with breast cancer and BRCA mutations, we have shown that mastectomy (versus lumpectomy) and mastectomy of the contralateral breast (versus unilateral mastectomy) were associated with large and statistically significant reductions in the risks of ipsilateral and contralateral breast cancer, respectively. ${ }^{23}$ We now show that the reduction in second primary cancers has a favourable impact on breast cancer specific mortality. In this cohort, the 20 year breast cancer specific mortality for women who had a unilateral mastectomy was $31 \%$; in the multivariate model, women with a mastectomy of the contralateral breast had a $48 \%$ reduction in risk of mortality compared with women with a unilateral mastectomy over a 20 year period.

We observed that the significant mortality benefit associated with mastectomy of the contralateral breast is most apparent in the second decade of follow-up after the initial diagnosis of breast cancer. The majority of deaths in the second decade of follow-up (55\%) occurred among women who experienced a second primary breast cancer. Our previous research has shown that women with a BRCA1 or BRCA2 mutation have a high risk of developing subsequent cancer in the contralateral breast. ${ }^{2}$ On average, the time from first breast cancer to ipsilateral breast cancer and breast cancer of the contralateral breast is 5.7 years. ${ }^{10}$ Therefore, a delay in the observed benefit of contralateral mastectomy is to be expected-that is, the reduction in mortality results from a reduction in deaths from cancer of the contralateral breast. To die from contralateral cancer, it is first necessary to be diagnosed with it and then to succumb to it. Furthermore, most women with a BRCA1 mutation and breast cancer have triple negative breast cancer (tumours that do not express oestrogen receptors, progesterone receptors, or human epidermal growth factor receptor $2 /$ neu protein),${ }^{11}$ and the majority of deaths from triple negative breast cancers occur in the first 10 years after diagnosis. ${ }^{12}$ The situation is perhaps analogous to that of radiation therapy and breast cancer mortality, wherein the benefit of radiotherapy in preventing death from breast cancer does not become apparent until the second decade after treatment. ${ }^{13}$ In the present study, a diagnosis of cancer in the contralateral breast was associated with a twofold increase in the rate of breast cancer specific mortality thereafter. It is critical, in the evaluation of contralateral mastectomy and breast cancer mortality, that the follow-up period is sufficiently long (for example, 20 years); in the short term, such as within five years of diagnosis, choice of contralateral mastectomy will be influenced by tumour stage and other prognostic factors, and almost all early deaths will be from the first primary cancer.

In the current study, we report that women with a BRCA1 (versus BRCA2) mutation were significantly more likely to die of their disease. However, the survival difference was not significant in the subgroup of cases that were treated with chemotherapy. This result is consistent with the conclusion of another study, which reported that carriers of the BRCA1 mutation had a worse prognosis than did carriers of the BRCA2 mutation or non-carriers, but only among women who did not receive chemotherapy. ${ }^{6}$ This may warrant the consideration of chemotherapy for all women with BRCA associated breast cancer. ${ }^{14}$

In the present study, $12 \%$ of the patients were initially treated with bilateral mastectomy, but $40 \%$ of the women treated with unilateral mastectomy opted for mastectomy of the contralateral breast at some time thereafter. This delay is most likely a result of women being unaware of their mutation status until after the 
initial surgery was completed. Among women who are aware that they have a mutation at the time of the initial surgery, the proportion who choose a bilateral mastectomy is much higher. ${ }^{15}$ In the future, if genetic testing is performed routinely at the time of diagnosis, comparisons of the various surgical treatments will become more straightforward, although long term follow-up data will not be available for decades.

\section{Strengths and limitations of this study}

The strengths of our study include the large sample size and the confirmation of all treatments by review of the medical records. All women with breast cancer in the families were identified and those who had had a diagnosis of breast cancer from 1975 to 2009 at age 65 or younger were eligible. We included untested women and deceased women in the study to avoid the survivorship bias that would arise if previous genetic testing was a condition for inclusion, but most of the participants (87\%) were confirmed carriers of a mutation. In the parent study, 720 women with breast cancer were tested for the family mutation; $691(96 \%)$ were found to carry the mutation and $29(4 \%)$ not to carry the mutation (and were excluded). This suggests that, of the 53 women included in this study who did not undergo genetic testing, we would expect that only two would be non-carriers - that is, less than $1 \%$ of the 390 participants in total.

The choice of surgical treatment for breast cancer was not randomised and several prognostic indicators in the treatment groups were imbalanced at baseline. We adjusted for BRCA mutation, tumour size, nodal status, and treatment factors in the survival analysis, but this is a relatively small study and it is possible that residual confounding may have influenced the results. In addition, we divided the follow-up period into two subperiods of one decade each; the reduction in mortality associated with mastectomy of the contralateral breast was much larger in the second decade than in the first $(80 \% v 35 \%)$, but many fewer deaths occurred in the second decade than in the first $(20 v 59)$. In the case of carriers of the BRCA1 and BRCA2 mutation, it is not practical to conduct randomised trials of the different surgical options because the carrier population is relatively small and because women often express strong preferences regarding their choice of treatment. We did not have access to information on progesterone receptor status or expression of human epidermal growth factor receptor 2 , and data on oestrogen receptor were missing for many participants, therefore we were unable to adjust for these three covariates. Furthermore, the data for the study were abstracted from the clinical chart notes and pathology reports and were not collected expressly for the purpose of this study. However, data from all centres was entered into a standardised coding form designed for this study. The notable finding in this study was a large reduction in breast cancer mortality in the second decade post-surgery, but this was based on only 20 deaths in this period.

We excluded from the study women initially treated with breast conserving surgery. This is because no patient had such surgery and mastectomy of the contralateral breast and therefore it would not be possible to distinguish the effects of ipsilateral mastectomy from contralateral mastectomy on survival. The study design allowed us to isolate and measure the effect of contralateral mastectomy on survival.

We assume that cancers in the contralateral breast represent second primary events and are not metastases. It is possible that several these contralateral cancers are in fact metastases, but if that were the case, then we would not expect contralateral mastectomy to prevent death from breast cancer. Also, in the event of a death of a woman after bilateral cancers, it was not possible to assign the cause of death to one or the other cancer.

\section{Conclusions}

We conclude that it is reasonable to propose bilateral mastectomy as the initial treatment option for women with early stage breast cancer who are carriers of a BRCA1 or BRCA2 mutation. For those who have been treated in the past with unilateral mastectomy or breast conserving surgery, the possibility of a second surgery should be discussed. It is important that our observations be confirmed in other study populations. These data, coupled with emerging data on the unique sensitivities of BRCA related breast cancers to certain classes of chemotherapy, ${ }^{16}{ }^{17}$ suggest that women with newly diagnosed breast cancer might benefit from the knowledge that they carry a BRCA mutation.

Contributors: KM and SAN conceived and planned the study and drafted the manuscript. SG collected and coordinated the data. PS performed all data analysis. HTL, PG, NT, CK-S, CS, AE, WDF, and BR coordinated research activities at their respective institutions. All authors read and approved the final manuscript. KM and SAN are the guarantors.

Funding: This research was funded by the Canadian Breast Cancer Foundation (Ontario Chapter). KM is supported by the Canadian Institutes of Health Research and the Ontario Women's Health Council. Competing interests: All authors have completed the ICMJE uniform disclosure form at www.icmje.org/coi_disclosure.pdf and declare: no support from any organisation for the submitted work; no financial relationships with any organisations that might have an interest in the submitted work in the previous three years; no other relationships or activities that could appear to have influenced the submitted work.

Ethical approval: This research was approved by the research ethics boards of all Womens College Research Institute (institutional review board approval No 2007-0075).

Data sharing: No additional data available.

Transparency: The lead author affirms that this manuscript is an honest, accurate and transparent account of the study being reported; that no important aspects of the study have been omitted; and that any discrepancies from the study as planned (and, if relevant, registered) have been explained.

1 Antoniou A, Pharoah PD, Narod S, Risch HA, Eyfjord JE, Hopper JL, et al. Average risks of breast and ovarian cancer associated with BRCA1 or BRCA2 mutations detected in case series unselected for family history: a combined analysis of 22 studies. Am J Hum Genet 2003;72:1117-30.

2 Metcalfe K, Lynch HT, Ghadirian P, Tung N, Olivotto I, Warner E, et al. Contralateral breast cancer in BRCA1 and BRCA2 mutation carriers. J Clin Oncol 2004;22:2328-35.

3 Metcalfe KA, Lubinski J, Ghadirian P, Lynch H, Kim-Sing C, Friedman E, et al. Predictors of contralateral prophylactic mastectomy in women with a BRCA1 or BRCA2 mutation: the Hereditary Breast Cancer Clinical Study Group. J Clin Oncol 2008;2:1093-7.

4 Narod SA. The impact of contralateral mastectomy on mortality in BRCA1 and BRCA2 mutation carriers with breast cancer. Breast Cancer Res Treat 2011;128:581-3.

5 Rennert G, Bisland-Naggan S, Barnett-Griness O, Bar-Joseph N, Zhang S, Rennert HS, et al. Clinical outcomes of breast cancer in carriers of BRCA1 and BRCA2 mutations. $N$ Engl J Med 2007;357:115-23.

6 Robson ME, Chappuis PO, Satagopan J, Wong N, Boyd J, Goffin JR, et al. A combined analysis of outcome following breast cancer: differences in survival based on BRCA1/BRCA2 mutation status and administration of adjuvant treatment. Breast Cancer Res 2004;6:R8-17.

7 Domchek SM, Friebel TM, Singer CF, Evans DG, Lynch HT, Isaacs C, et al. Association of risk-reducing surgery in BRCA1 or BRCA2 mutation carriers with cancer risk and mortality. JAMA 2010;304:967-75.

8 Goodwin PJ, Phillips KA, West DW, Ennis M, Hopper JL, John EM, et al. Breast cancer prognosis in BRCA1 and BRCA2 mutation carriers: an international prospective breast cancer family registry population-based cohort study. J Clin Oncol 2012;30:19-26.

9 Lostumbo L, Carbine NE, Wallace J. Prophylactic mastectomy for the prevention of breast cancer. Cochrane Database Syst Rev 2010;11:CD002748.

10 Metcalfe K, Gershman S, Lynch HT, Ghadirian P, Tung N, Kim-Sing C, et al. Predictors of contralateral breast cancer in BRCA1 and BRCA2 mutation carriers. Br J Cancer 2011;104:1384-92.

11 Foulkes WD, Stefansson IM, Chappuis PO, Bégin LR, Goffin JR, Wong N, et al. Germline BRCA1 mutations and a basal epithelial phenotype in breast cancer. J Natl Cancer Inst 2003;95:1482-5. 


\section{What is already known on this topic}

Women who carry a germline mutation in either the BRCA1 or BRCA2 gene have a $60 \%$ risk of breast cancer and once diagnosed a $34 \%$ risk of cancer in the contralateral breast by 15 years

Mastectomy of the contralateral breast is associated with a large reduction in the risk of contralateral breast cancer, but it has not yet been shown that contralateral mastectomy reduces breast cancer related mortality

\section{What this study adds}

In this non-randomised observational study, women with BRCA associated breast cancer who were treated with bilateral mastectomy were $48 \%$ less likely to die of breast cancer within 20 years of diagnosis than women treated with unilateral mastectomy

Bilateral mastectomy should be discussed as an option for young women with a BRCA mutation and early onset breast cancer

Given the small number of events in this cohort, further research is required to confirm these findings

12 Dent R, Trudeau M, Pritchard KI, Hanna WM, Kahn HK, Sawka CA, et al. Triple-negative breast cancer: clinical features and patterns of recurrence. Clin Cancer Res 2007;13:4429-34.

13 Early Breast Cancer Trialists Collaborative Group. Effects of radiotherapy and of differences in the extent of surgery for early breast cancer on local recurrence and 15-year survival: an overview of the randomised trials. Lancet 2005:366:2087-106.

14 Narod SA, Metcalfe K, Lynch HT, Ghadirian P, Robidoux A, Tung N, et al. Should all BRCA1 mutation carriers with stage i breast cancer receive chemotherapy? Breast Cancer Res Treat 2013;138:273-9.

15 Weitzel JN, McCaffrey SM, Nedelcu R, MacDonald DJ, Blazer KR, Cullinane CA. Effect of genetic cancer risk assessment on surgical decisions at breast cancer diagnosis. Arch Surg 2003;138:1323-8.

16 Byrski T, Gronwald J, Huzarski T, Grzybowska E, Budryk M, Stawicka M, et al. Pathological complete response rates in young women with BRCA1-positive breast cancers after neoadjuvant chemotherapy. J Clin Oncol 2010;28:375-9.
17 Byrski T, Dent R, Blecharz P, Foszczynska-Kloda M, Gronwald J, Huzarski T, et al. Results of a phase II open-label, non randomized trial of cisplatin chemotherapy in patients with BRCA1-positive metastatic breast cancer. Breast Cancer Res 2012;14:R110.

\section{Cite this as: BMJ 2014;348:g226}

This is an Open Access article distributed in accordance with the Creative Commons Attribution Non Commercial (CC BY-NC 3.0) license, which permits others to distribute, remix, adapt, build upon this work non-commercially, and license their derivative works on different terms, provided the original work is properly cited and the use is non-commercial. See: http://creativecommons.org/licenses/by-nc/3.0/. 


\section{Tables}

Table 1 | Characteristics of 390 women with breast cancer and details of their treatment. Values are numbers (percentages) unless stated otherwise

\begin{tabular}{|c|c|}
\hline Characteristics & Values \\
\hline Year of birth (range) & $1947(1914-75)$ \\
\hline Mean (range) duration of follow-up (years) & $13.0(0.1-20.0)$ \\
\hline \multicolumn{2}{|l|}{ From diagnosis: } \\
\hline Mean (range) years alive & $14.5(0.1-20.0)$ \\
\hline Mean (range) years to death & $7.1(0.7-19.3)$ \\
\hline Mean (range) age at diagnosis & $42.7(22.0-65.0)$ \\
\hline \multicolumn{2}{|l|}{ Age group: } \\
\hline$<30$ & $21(5.4)$ \\
\hline 30-39 & $144(36.9)$ \\
\hline $40-49$ & $139(35.6)$ \\
\hline$\geq 50$ & $86(22.0)$ \\
\hline \multicolumn{2}{|l|}{ Mutation: } \\
\hline BRCA1 & $226(57.9)$ \\
\hline BRCA2 & $158(40.2)$ \\
\hline Both BRCA1 and BRCA2 & $6(1.5)$ \\
\hline \multicolumn{2}{|l|}{ Tumour size $(\mathrm{cm})$ : } \\
\hline $0-2.0$ & $228(60.2)$ \\
\hline $2.1-5.0$ & $151(39.8)$ \\
\hline Unknown & 11 \\
\hline Mean (range) & $2.1(0.1-5.0)$ \\
\hline \multicolumn{2}{|l|}{ Oestrogen receptor status: } \\
\hline Positive & $127(49.4)$ \\
\hline Negative & $130(50.6)$ \\
\hline Unknown & 133 \\
\hline \multicolumn{2}{|l|}{ Nodal status: } \\
\hline Positive & $159(41.0)$ \\
\hline Negative & $229(59.0)$ \\
\hline Unknown & 2 \\
\hline \multicolumn{2}{|l|}{ Histology } \\
\hline Medullary & $31(8.0)$ \\
\hline Ductal & $319(81.8)$ \\
\hline Lobular & $18(4.6)$ \\
\hline Other/unknown & $22(5.6)$ \\
\hline \multicolumn{2}{|l|}{ Initial surgery: } \\
\hline Unilateral mastectomy & $346(88.7)$ \\
\hline Bilateral mastectomy & $44(11.3)$ \\
\hline \multicolumn{2}{|l|}{ Contralateral mastectomy: } \\
\hline No & 209 \\
\hline At initial surgery & 44 \\
\hline After initial surgery & 137 \\
\hline \multicolumn{2}{|l|}{ Chemotherapy: } \\
\hline Yes & $236(62.6)$ \\
\hline No & $141(37.4)$ \\
\hline Missing & 13 \\
\hline
\end{tabular}


Table 1 (continued)

\begin{tabular}{lc} 
Characteristics & Values \\
Yes & $71(18.6)$ \\
\hline No & $310(81.4)$ \\
\hline Missing & 9 \\
\hline Tamoxifen: & $111(30.7)$ \\
\hline Yes & $251(69.3)$ \\
\hline No & 28 \\
\hline Missing & $156(40.7)$ \\
\hline Oophorectomy: & $227(59.3)$ \\
\hline Never & 7 \\
\hline Ever &
\end{tabular}


Table 2| Comparison of participants who underwent initial unilateral mastectomy or bilateral mastectomy. Values are numbers (percentages) unless stated otherwise

\begin{tabular}{lccc} 
Characteristics & Unilateral mastectomy $(\mathbf{n = 2 0 9 )}$ Bilateral mastectomy $(\mathbf{n = 1 8 1})$ & P value \\
Age at diagnosis (years) & 43.6 & 41.3 & 0.01 \\
\hline Year of diagnosis & 1987 & 1994 & $<10$ to 4 \\
\hline Size of tumour $(\mathrm{cm}):$ & & & \\
\hline $0-2$ & $114(55.9)$ & $114(65.1)$ & 0.07 \\
\hline $2.1-5$ & $90(44.1)$ & $61(34.9)$ & 0.006 \\
\hline Mean (range) size & $2.3(0.2-5.0)$ & $1.9(0.1-5.0)$ & 0.39 \\
\hline Positive lymph nodes & $89(43.0)$ & $70(38.7)$ & 0.03 \\
\hline Chemotherapy & $115(57.5)$ & $121(68.4)$ & $<10$ to 4 \\
\hline Oophorectomy & $97(48.0)$ & $130(71.8)$ & 0.06 \\
\hline Tamoxifen & $51(26.5)$ & $60(35.5)$ & $<10$ to 4 \\
\hline Contralateral breast cancer & $70(33.5)$ & $1(0.6)$ & $<10$ to 4 \\
\hline Died from breast cancer & $61(29.2)$ & $18(9.9)$ & 0.33 \\
\hline Radiotherapy & $41(20.5)$ & $30(16.6)$ & 0.63 \\
\hline BRCA1 & $123(60.0)$ & $103(57.5)$ & $76(42.5)$ \\
\hline BRCA2 & $82(40.0)$ & &
\end{tabular}


Table 3| Hazard ratios for breast cancer related death associated with contralateral mastectomy versus no contralateral mastectomy

\begin{tabular}{|c|c|c|c|c|c|c|}
\hline Follow-up (years) & $\begin{array}{l}\text { Univariate hazard ratio } \\
\qquad(95 \% \mathrm{Cl})\end{array}$ & $P$ value & $\begin{array}{l}\text { Multivariate* hazard ratio } \\
\qquad(95 \% \mathrm{Cl})\end{array}$ & $P$ value & $\begin{array}{l}\text { Matched† hazard ratio } \\
\qquad(95 \% \mathrm{Cl})\end{array}$ & $P$ value \\
\hline $0-20$ & 0.48 (0.29 to 0.82$)$ & 0.007 & 0.52 (0.29 to 0.93$)$ & 0.03 & $0.60(0.34$ to 1.06$)$ & 0.08 \\
\hline $0-10$ & $0.60(0.34$ to 1.06$)$ & 0.08 & 0.65 (0.34 to 1.22$)$ & 0.18 & $0.72(0.39$ to 1.33$)$ & 0.29 \\
\hline $10-20$ & $0.20(0.05$ to 0.85$)$ & 0.03 & 0.20 (0.05 to 0.89$)$ & 0.03 & 0.26 (0.06 to 1.15$)$ & 0.08 \\
\hline
\end{tabular}

*Adjusted for BRCA mutation, tumour size, nodal status, age at diagnosis, year of diagnosis, chemotherapy (yes or no), radiotherapy, and oophorectomy (time dependent variable).

†Matched by propensity score; total of 79 matched pairs analysed. 
Table 4| Hazard ratios for death associated with non-surgical treatments

\begin{tabular}{lcccc} 
Variables & Univariate hazard ratio $(95 \% \mathrm{Cl})$ & $\mathbf{P}$ value & Multivariate* hazard ratio $(95 \% \mathrm{Cl})$ & P value \\
\hline Tumour size $>2 \mathrm{~cm} v \leq 2 \mathrm{~cm}$ & $1.66(1.05$ to 2.61$)$ & 0.03 & $1.46(0.89$ to 2.38$)$ & 0.13 \\
\hline Nodal status positive $v$ negative & $1.70(1.09$ to 2.65$)$ & 0.02 & $2.32(1.26$ to 4.28$)$ & 0.007 \\
\hline Chemotherapy yes $v$ no & $1.13(0.71$ to 1.80$)$ & 0.62 & $0.57(0.30$ to 1.09$)$ & 0.09 \\
\hline Radiotherapy yes $v$ no & $1.62(0.95$ to 2.76$)$ & 0.07 & $1.61(0.91$ to 2.84$)$ & 0.10 \\
\hline Tamoxifen yes $v$ no & $1.00(0.60$ to 1.66$)$ & 0.99 & $1.18(0.65$ to 2.16$)$ & 0.58 \\
\hline Oophorectomy yes $v$ no & $0.78(0.47$ to 1.28$)$ & 0.32 & $0.67(0.38$ to 1.17$)$ & 0.16 \\
\hline BRCA2 $v$ BRCA1 & $0.64(0.40$ to 1.04$)$ & 0.07 & $0.45(0.26$ to 0.80$)$ & 0.006 \\
\hline
\end{tabular}

${ }^{*}$ Adjusted for all variables in table plus age at diagnosis and year of diagnosis. †Time dependent covariate. 


\section{Figures}

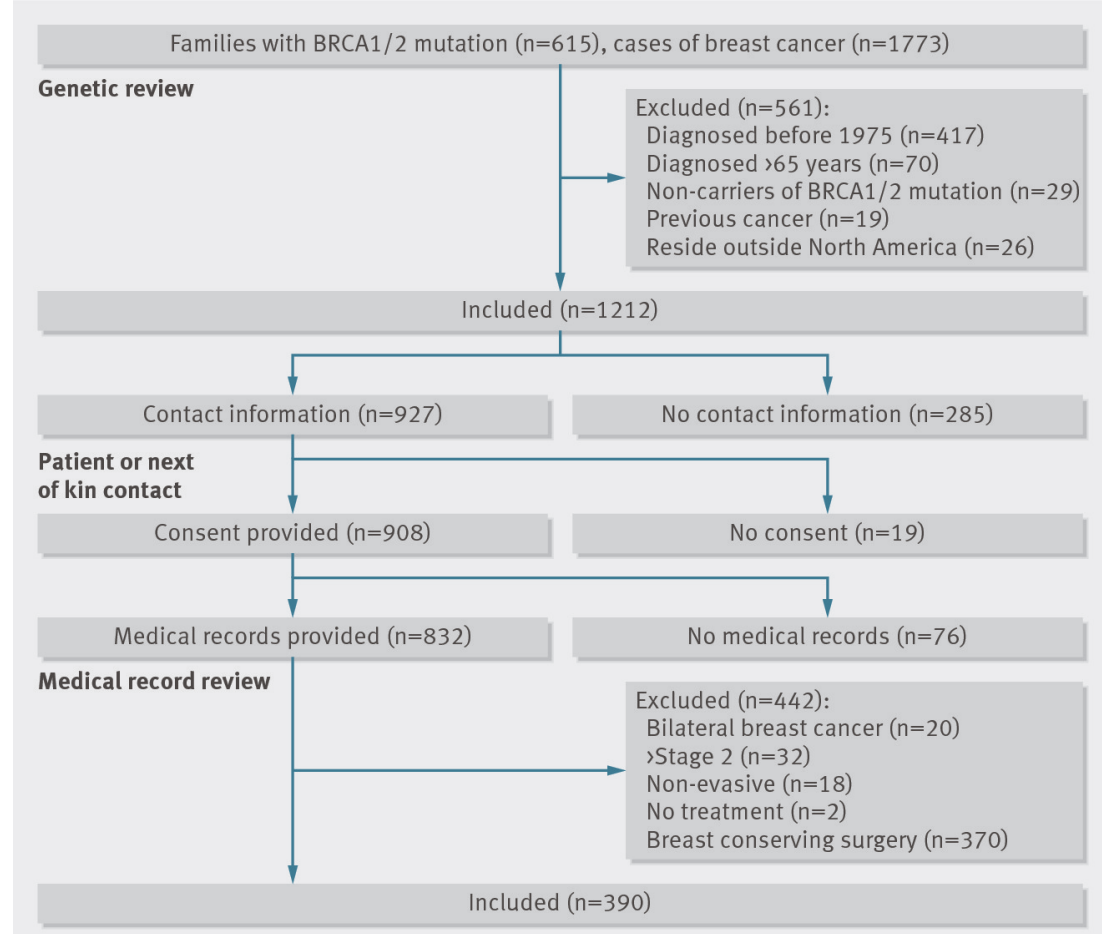

Fig 1 Flow of participants through study

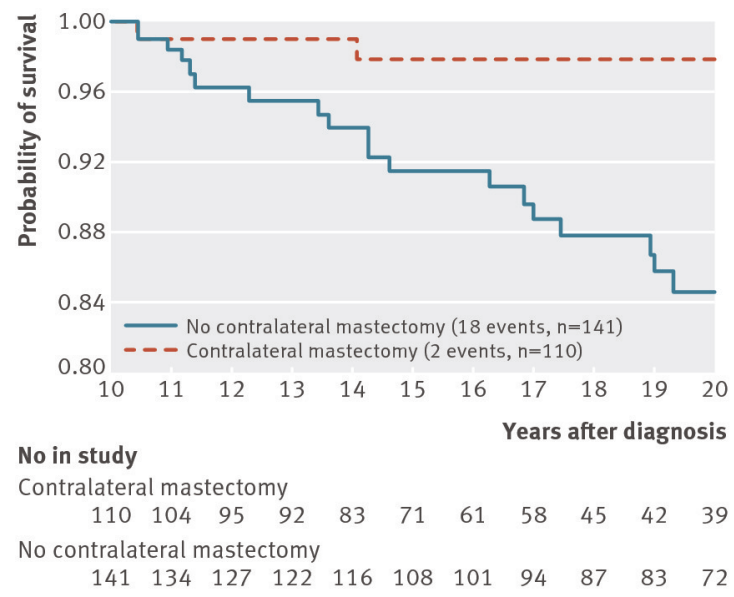

Fig 2 Survival from 10 to 20 years after breast cancer, by contralateral mastectomy 